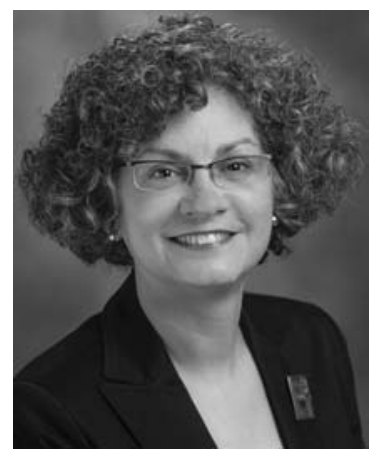

\title{
A Conversation with Guillermina (Gigi) Lozano
}

\author{
InTERVIEwer: Gemma Alderton \\ Senior Editor, Nature Reviews Cancer
}

Guillermina Lozano is Chair of the Department of Genetics at the University of Texas MD Anderson Cancer Center.

Gemma Alderton: Could you give us a bit of background about where we're at in the field of p53 and what's happening with regard to mutations of $\mathrm{p} 53$ ?

Dr. Lozano: All the new, next-generation sequencing data that has been generated over the last few years has basically said that p53 is mutated in almost every cancer that you can think of. We've been working with p53 mutations in animal models, trying to understand how mutant p53 converts a normal, perhaps preneoplastic, cell into something that becomes a very aggressive cell. Mutations in p53 are very common, and these mutations lead to very aggressive tumor types. We've developed some of the animal models that allow us to examine the functions of p53 in vivo.

p53 is a tetramer that binds directly to DNA and activates transcription of probably several hundred genes. There's also a lot of tissue specificity. For example, p53 will activate the p21 cell cycle inhibitor 200 -fold in one tissue, but only twofold in another. There's still a lot to learn about its function as a transcription factor. The fascinating part about p53 is the kinds of mutations that occur in human cancers. Most of these mutations occur in the DNA binding domain. Basically, they disrupt the ability of $\mathrm{p} 53$ to function as a transcription factor.

Gemma Alderton: These mutations can be loss-of-function or gain-of-function, right?

Dr. Lozano: Yes. There are a few mutations that are truncating mutations, but depending on the tumor type, $70 \%-80 \%$ are missense mutations. Years ago, there was a lot of talk about those missense mutations and why it was so common to have a p53 mutation. One idea was that it was very easy to make a missense mutation in a genomic region. I think the real reason is because these mutant p53 proteins give the cell a growth advantage. The cell becomes able to cope with other changes and they evolve more quickly when it has a missense mutation versus no p53 protein.

Gemma Alderton: Do you see these mutations in specific types of cancer, or is it very broad?

Dr. Lozano: It's very broad. There are five hotspots, all arginines. Four of those arginines directly contact the DNA, so it makes sense that those are common mutations. The other hotspot basically disrupts the whole structure of the DNA binding domain. There are a few differences in different tissues but not enough to really know if there are differences between the kinds of mutations. We've been able to mimic these mutations in the mouse, and we're beginning to see some subtle differences. I think the models will be able to tell us if there is a difference in how these mutations lead to aggressive breast tumors, or lung tumors.

Gemma Alderton: Does the p53 mutation have more to do with initiation or tumor progression?

Dr. Lozano: In ovarian cancer, it's definitely the first mutation that happens. It is very early, and about $95 \%$ of high-grade serous ovarian cancers have p53 mutations. Some clinicians suggest that it's really $100 \%$, and that the few percent without p53 mutations are just misdiagnosed tumors. On the other hand, if you look at colorectal cancer, it's the last mutation; it's what takes the lesion from staying in the colon to becoming a metastatic lesion. These are two very different scenarios. In both cases though, once that p53 is mutant (and on top of that, it has to become a stable mutant p53 by signals that the tumor sends out), it contributes to a very aggressive cancer. Timing is important, but it varies between the different cancers.

Gemma Alderton: Are different mechanisms involved depending on whether mutation happens early or late?

(C) 2016 Lozano. This article is distributed under the terms of the Creative Commons Attribution-NonCommercial License, which permits reuse and redistribution, except for commercial purposes, provided that the original author and source are credited. 
Dr. Lozano: There are two answers to your question: One is what happens upstream and when. From studies in animal models, we know that mutant p53 protein is not very stable, in contrast to what we'd thought for years. In most tumors, there are some signals - stress signals, inflammatory signals - that lead to stable mutant protein. Once you have a stable mutant protein, you can have these additional activities, this more aggressive cancer. It's really a two-step process. Perhaps those signals make the mutant stable because the cell is stressed and to proliferate it has to mutate $\mathrm{p} 53$, but to progress that mutant protein has to be stabilized.

The second part of the answer involves what these mutant proteins do after they become stable. They bind to numerous proteins, and a lot of them are transcription factors. In those cases, the mutant $\mathrm{p} 53$, which has a very potent transcription activation domain, is being dragged to other promoters and is allowing expression of genes the cell would never see normally. Which of those proteins a mutant p53 interacts with will be different in different tissues and will also depend on what else is altered in the cell.

Gemma Alderton: p53 is traditionally associated with regulating processes like metabolism and DNA damage responses. Does mutant p53 serve other roles?

Dr. Lozano: Mutant p53 does a lot of things. Carol Prives published studies showing it affects the sterol synthesis pathway, but we still have to figure out what it means physiologically. If affects integrin recycling (this is from Karen Vousden's laboratory) and the vitamin D receptor (Varda Rotter's laboratory). The field knows that there are a lot of things that mutant p53 does. We need to move from the tissue culture systems where most of these assays have been performed to in vivo systems to ask which mutants, and targets, and pathways it affects.

Perhaps instead of looking at specific genes as markers for the evolution of tumors, we should look at biological processes, pathways, or stress signals. Instead of putting all our hopes into a single gene or gene product to characterize the cancer, we need to really understand the biology of that cancer and how it evolved and how it got to where it is. p53 certainly has a role, but we still have to figure out exactly what that role is. All we really know is that if the tumor cell has a stable mutant p53 protein, it's a much more aggressive tumor.

Gemma Alderton: Where are we at in terms of understanding the role of p53 in promoting metastasis?

Dr. Lozano: The models that Tyler Jacks made a number of years ago basically said that a cell with a mutant p53 protein expressed at high levels is going to be highly metastatic. We're beginning to understand that evolution. The problem is that we currently only have two types of models. In the first, the p53 is mutant everywhere, so the mice develop all kinds of tumors spontaneously. It's very difficult to just study breast cancer, because you get lymphomas, you get sarcomas. Just one model will give you so many different kinds of cancers.
The other model starts out null for p53, so the whole mouse is heterozygous for $\mathrm{p} 53$. You can then have a p53 mutation in a single cell, or a single tissue, or organ. That helps a bit because now the mutation is only in a single cell type, but the immune system and stroma is heterozygous for p53. There's quite a bit of data that say that the stroma affects how a tumor develops. One of the new alleles that we made is one in which we can make a mutant p53 basically in one single cell surrounded by a completely normal stroma and immune system. That allows us to do a whole bunch of things. We're still in the early stages, but we're beginning to get some tumors one to two years later. We weren't even sure we were going to get a tumor because we were making p53 mutations in such a small number of cells. What if we needed to hit 10 million cells before we could get a tumor? We're really into uncharted territory.

The other thing that we're doing is that when we mutate the cells - it's a Cre-mediated recombination processwe can label cells with the p53 mutation with red fluorescence. Now you can follow circulating tumor cells; you can actually see a tiny little metastasis. We're hoping that we can examine tumor dormancy in tissues. It's still early, but it's the first time that we've been able to label a mutant p53 cell in a completely normal environment.

Gemma Alderton: You mentioned that you could extract circulating tumor cells from these mice.

Dr. Lozano: It's a very small number, but they're present in some of these models. Comparison of the primary tumor versus what's circulating versus what targets the bone or the liver is going to be really helpful. Metastasis is such a complicated process. We've learned so much from our tissue culture systems, but we need to take that next step and go to physiological systems where we're actually looking at the entire process and the whole organism.

Gemma Alderton: The tumor marker environment is extraordinarily important for metastases. What do we know about p53's role in the stroma?

Dr. Lozano: There's one study published a number of years ago by Terry Van Dyke that looked at the development of prostate cancer. Because of the alleles that we had at the time, the stroma in her model was p53 heterozygous. The stroma and the tumors interacted, and that tumor evolved very rapidly, but the stroma was losing that other p53 allele so that the tumor could get nutrients or something from the stroma. That is one of the best examples showing that what happens in the stroma with regard to p53 affects what happens in the tumor. We're doing similar experiments now in our new model, but it takes time. First, we had to characterize the model to make sure it worked. We can now take out p53 just in the stroma, and look at, say, a Wnt tumor in the breast or another kind of tumor and see how it develops in this environment.

The mouse models are fascinating. I started in the field because I went to interview for a postdoc with Arnie Levine and he said we can manipulate the mouse and 
make a tumor model. That's what really drew me to the field - that you can make a point mutation in the mouse and then study the effects of that mutation. Now with the new CRISPR technologies, we can do so much more.

Gemma Alderton: p53 is part of a family of proteins that are also mutated in cancer.

Dr. Lozano: The two other family members are p63 and $\mathrm{p} 73$. There are very few mutations in those proteins in cancers. I'm convinced that p63 has a role in epithelial cancers, but it's usually downmodulated. There's some data that p73 is also downmodulated, but nowhere near the level of p53. I do think they have a role, but it's probably minor.

Gemma Alderton: What are the key challenges in your work going forward?

Dr. Lozano: Ute Moll's laboratory recently published data showing that the lymphoma she was studying in an animal model is "addicted" to having a mutant p53. If you take out that mutant $\mathrm{p} 53$, the tumor basically can't deal with it. That's one direction that the field has to go in. If you have a tyrosine kinase expressed at high levels, the drugs you tend to use are aimed at inhibiting this activity.
Here you have to reactivate p53, to put it back into the system. That's hard. How do you do that?

If Moll's results turn out to be more broadly applicable to other kinds of cancers, we should be thinking about drugs that disrupt mutant p53 interactions with other proteins that affect metastasis. We're not going to cure cancer. Hopefully, we can make it a stable disease where we can understand enough about what the tumor wants or requires to grow that it can be stuck there in its own little environment and not grow. We need to think more broadly about how to target p53. There are some drugs in the clinic right now that disrupt the interaction between p53 and an important negative regulator called Mdm2, which is an E3 ligase that targets p53 for degradation. In many tumors, you'll have p53 mutations, whereas in others you'll have amplification of Mdm2 at very high levels. Drugs targeting Mdm2 have been around for a long time; some are in clinical studies with mixed results right now. These drugs can disrupt the p53/Mdm2 interaction, but I think you also have to keep these two proteins apart for a longer time. I think the drugs just create a seesaw effect where p53 isn't constantly on, and so you can't tip the balance.

Thanks, Gemma. I enjoyed talking to you. 


\section{$\$_{\text {CSH\& }}^{\infty}$ Cold Spring Harbor Symposia SYMPOSIA}

\section{A Conversation with Guillermina (Gigi) Lozano}

Cold Spring Harb Symp Quant Biol 2016 81: 323-325 originally published online December 8, 2016

Access the most recent version at doi:10.1101/sqb.2016.81.031187
Creative This article is distributed under the terms of the
Commons http://creativecommons.org/licenses/by-nc/4.0/, which permits reuse and
License redistribution, except for commercial purposes, provided that the original author and source are credited.

Email Alerting Receive free email alerts when new articles cite this article - sign up in Service the box at the top right corner of the article or click here. 